Théologiques

Théologiques

\title{
Traditions et principes de la traduction biblique dans l'Antiquité juive
}

\section{Francine Kaufmann}

Volume 15, numéro 2, 2007

La traduction des textes sacrés

URI : https://id.erudit.org/iderudit/017771ar

DOI : https://doi.org/10.7202/017771ar

Aller au sommaire du numéro

\section{Éditeur(s)}

Faculté de théologie et de sciences des religions, Université de Montréal

\section{ISSN}

1188-7109 (imprimé)

1492-1413 (numérique)

Découvrir la revue

Citer cet article

Kaufmann, F. (2007). Traditions et principes de la traduction biblique dans l'Antiquité juive. Théologiques, 15(2), 15-45. https://doi.org/10.7202/017771ar
Résumé de l'article

Un préjugé répandu voudrait qu'un tabou juif interdise de traduire la Bible ou n'autorise qu'une traduction servile, littérale. Cette étude s'appuie sur la littérature rabbinique (Talmud, Midrach, exégèse) pour montrer qu'une traduction orale à usage interne a été prescrite dès l'Antiquité, quand la majorité des Juifs ont perdu l'usage de l'hébreu. Sa fonction était d'accompagner - non de remplacer - l'original hébraïque, dans la lecture synagogale et dans l'étude. Elle a été mise plus tard par écrit puis imprimée en regard de l'original. La traduction qui s'enracine dans la tradition exégétique juive est légitime puisqu'elle contribue à clarifier et expliquer et le sens de la Bible hébraïque.
Tous droits réservés (C) Faculté de théologie et de sciences des religions, Université de Montréal, 2007
Ce document est protégé par la loi sur le droit d'auteur. L’utilisation des services d'Érudit (y compris la reproduction) est assujettie à sa politique d'utilisation que vous pouvez consulter en ligne.

https://apropos.erudit.org/fr/usagers/politique-dutilisation/ 


\title{
Traditions et principes de la traduction biblique dans l'Antiquité juive
}

\author{
Francine KAUFMANN \\ Département de traduction \\ Université Bar Ilan — Israël
}

\section{Avant-propos: les clichés concernant la traduction juive de la Bible}

Bien des clichés accompagnent les jugements sur l'approche juive de la traduction biblique. L'un d'eux voudrait que le judaïsme condamne toute traduction de la Bible. (Il est vrai que les juifs ${ }^{1}$ orthodoxes jeûnent aujourd'hui encore, le 10 du mois de Téveth, pour marquer l'anniversaire de l'achèvement de la Septante). Par ailleurs, la théologie chrétienne a longtemps présenté les juifs comme des adorateurs de la lettre, qui ne se résolvent à la traduction qu'en sacrifiant l'esprit au respect aveugle et minutieux de la littéralité. On pense encore trop souvent aujourd'hui que, par peur de s'écarter d'un iota du texte écrit canonisé, une traduction juive de la Bible,

1. Nous respectons, dans cet article, les conventions d'écriture de la langue française: nous écrivons ainsi «les juifs ", sans majuscule, chaque fois qu'il s'agit des adeptes de la religion juive, la majuscule indiquant l'appartenance au peuple juif : les Juifs. Pour les citations bibliques, nous emploierons le système d'abréviation de la BJ (Bible de Jérusalem, éd. du Cerf). Nous adoptons, pour les citations du Talmud les conventions suivantes $: \mathrm{TB}=$ Talmud de Babylone, $\mathrm{TJ}$ = Talmud de Jérusalem, suivi du nom en italiques du traité concerné, puis du numéro du feuillet selon l'édition hébraïque consacrée de la veuve Romm auquel est accolée la lettre a (recto) ou b (verso). La Michna est citée selon le chapitre et le numéro de la michna concernée. Chaque recueil du Midrach porte un nom (en italiques) et, soit le numéro cardinal de la page, soit le nom de la section sabbatique auquel il se rapporte. Pour les transcriptions de l'hébreu, parmi les très nombreux systèmes prévalant souvent de manière anarchique, nous avons privilégié une tradition juive française, proche de la prononciation de l'hébreu: ch (et non sh) désigne ainsi le son ch de 'chat' et non le son allemand contenu dans 'Buch'. Le y transcrit le yod, le h rend le hey et le ' $h$ le 'hète. Kh renvoie au khaf, $k$ au kaf et qu au kouf. Pour le son ou nous écrivons ou et non u (ex: Yehouda) et conservons la forme hébraïque des noms propres cités dans les sources juives (comme cela se fait couramment aujourd'hui dans la plupart des maisons d'édition), sauf pour les formes francisées passées dans l'usage courant. 
lorsqu'elle existe, pratique systématiquement le littéralisme, reproduisant autant que faire se peut la forme de l'original (syntaxe, ordre des mots, sens étymologique, etc.). En contrepoint, on a parallèlement considéré les juifs comme des faussaires, des falsificateurs du texte biblique, qu'ils auraient altéré, manipulé pour en extirper toute allusion préfigurant la venue du Christ-Messie, masquant et obscurcissant à souhait le message révélé pour se réserver à eux seuls l'accès des secrets divins.

Les traductologues (spécialistes des sciences de la traduction), héritiers de ces préjugés, évoquent dans leur sillage l'influence juive pour expliquer les résistances que suscita la traduction en Occident. Antoine Berman, dans la conclusion d'un essai sur les liens entre culture et traduction, postule que l'Europe a posé «l'intraduisible comme valeur» (notamment en poésie):

L'essentiel d'un texte n'est pas traduisible ou, à supposer qu'il le soit, il ne doit pas être traduit. Dans le cas de la Bible, c'est la tradition juive qui représente cette position extrême. Tout comme la «Loi» ne doit pas être «traduite " de l'oral à l'écrit, le texte sacré ne doit pas être traduit dans d'autres langues, sous peine de perdre son caractère «sacré» (Berman 1984, 298).

Ailleurs, il affirme: "Alors que la tradition juive se méfiait de la traduction, c'est vraiment un impératif catégorique du christianisme que la traduction du Livre dans toutes les langues, afin que le souffle vivifiant de l'Esprit atteigne toutes les nations" (Berman 1985, 52). Nombre de traductologues partent même du postulat qu'un tabou juif frappait la traduction de la parole divine. Ils s'appuient sur le texte désormais classique de Georges Steiner dans Après Babel, repris en version française par l'historien de la traduction Michel Ballard (De Cicéron à Benjamin, 1992, 38) : "Le judaïsme recèle un tabou encore plus extrême. Le [sic] Meguillath Taanith, qu'on estime remonter au premier siècle, rapporte que le monde s'obscurcit pendant trois jours quand la Loi fut traduite en $\operatorname{grec}^{2} »$. Un ouvrage récent de Douglas Robinson, Translation and Taboo (1996), qui s'inspire largement des concepts introduits par Steiner concernant la traduction

2. «An even more definite taboo can be found in Judaism. The Megillath Taanith (Roll of Fasting), which is assigned to the first century A.D., records the belief that three days of utter darkness fell on the world when the Law was translated into Greek » (Steiner 1975, 239). Michel Ballard, après avoir cité Steiner, conclut son développement sur la traduction biblique dans le monde juif par ces phrases: "Il y a donc dans la traduction un caractère transgressant puisqu'elle va à l'encontre d'un interdit de communication, puisqu'elle prétend véhiculer un sens, en principe inaccessible ou trop complexe, ou trop dépendant de ses formes pour ne pas être trahi par tout transfert. » (Ballard 1992, 38) 
sacrée en général et la traduction juive en particulier (cf. préface, p. xii-xiii), continue de propager les clichés qui affirment que le judaïsme rabbinique, puis la tradition kabbalistique, établissent l'impossibilité — voire interdisent — de traduire la Bible (cf. Steiner 1975, 61 et Robinson 1996, xiii, xvxvi, 64-70). Robinson étaye son argumentation en mettant en avant la croyance mystique (qu'il dit présente également dans le soufisme islamique) selon laquelle, de même que le corps et l'âme sont indissociables, de même la forme et le fond de la parole divine ne peuvent être séparés. Dans cette optique, affirme-t-il, seul l'original est sacré (ibid. 65).

Pour notre part, nous considérons que cette approche est biaisée et qu'elle doit, pour le moins, être fortement nuancée. Ainsi, la Michna (Meguilla 1,8) affirme que les Livres bibliques peuvent être traduits dans n'importe quelle langue, ou du moins en grec selon l'opinion restrictive de Rabban Siméon ben Gamliel (qui sera retenue dans la Guemara). Le Talmud permet d'enfreindre les lois du Chabbat pour sauver d'un incendie des livres sacrés en traduction. Un texte biblique traduit ne doit pas être détruit s'il est abîmé mais enterré, comme on le fait pour l'original hébraïque (TB Chabbat 115a-b). En ce qui concerne la référence de Steiner à Meguillath Taanith (littéralement: le rouleau des Jeûnes), nous avons vérifié et constaté que ce recueil ne contient pas la liste des jours de jeûne, mais au contraire celle de tous les jours de fête commémorant d'heureux événements. Il constitue donc une liste des jours où il est interdit de jeûner. Ce rouleau rédigé en araméen, sans doute au $\mathrm{I}^{\mathrm{er}}$ siècle, est clos par cette formule: "Ainsi s'achève la Meguillath Taanith». Dans sa forme actuelle, il contient un addendum en hébreu (et non plus en araméen), désigné comme: Maamar a'harone (Postface) qui, de l'avis des savants, daterait du VIII siècle. C'est là que figure cette phrase: «Le 8 du mois de Teveth, la Torah fut traduite en grec sous le roi Ptolémée. Et les ténèbres couvrirent le monde pendant trois jours ${ }^{3} »$ (Meguillath Taanith 13, nous traduisons). À cette époque, les débats théologiques entre le judaïsme et les religions issues d'elles (christianisme et islam) avaient transformé l'interprétation du texte biblique en arme aux effets lourds de conséquences dans les relations entre les peuples, comme nous le verrons plus loin. Ce prétendu «tabou»

3. Soulignons le lien entre cette formule et la neuvième plaie d'Egypte: «Moïse étendit sa main vers le ciel; et il y eut d'épaisses ténèbres dans tout le pays d'Égypte, pendant trois jours. " (Ex 10,22) Notons que l'Egypte est le pays lié à la traduction de la LXX. Le jeûne du 10 Teveth est placé au troisième jour des ténèbres qui s'abattirent le 8 Teveth. 
n'empêcha pas au X $X^{\mathrm{e}}$ siècle Sa'adia Gaon, rabbin, exégète et grammairien ${ }^{4}$, de traduire une grande partie de la Bible hébraïque et de la commenter en arabe (essentiellement en réaction à l'hérésie juive karaïte qui prônait une interprétation littéraliste de la Bible, mais aussi dans un dialogue avec le milieu arabe puisque sa traduction était écrite en caractères arabes). Son Tafsir fut introduit dans le rituel synagogal des juifs d'Afrique du nord (cf. Chouraqui 1972, 91) et du Yémen, en guise de targoum.

Des traductions juives ont fleuri à travers l'histoire, dans toutes les langues de l'exil, la langue vernaculaire étant, il est vrai, généralement transcrite dans l'alphabet hébraïque, ce qui indiquait une intention "centripète ", une traduction à usage interne, tout comme dans le cas des traductions en judéo-langues (ladino, yiddish, judéo-arabe, etc.). Se pourrait-il néanmoins que la réalité ait été en contradiction avec la position des rabbins en matière de traduction ou la position des rabbins serait-elle plus « ouverte » à la traduction biblique qu'on ne le suppose généralement ? Enfin, que disaient de la traduction les rabbins, avant que des débats idéologiques ne les opposent à des adversaires dans le domaine de la foi ?

Le cadre de cet article ne nous permet pas de traiter exhaustivement de ce thème complexe et infiniment riche, à travers le temps et l'espace. Nous avons d'ailleurs déjà abordé certains aspects de l'approche juive de la traduction dans des articles antérieurs (Kaufmann 1990, 1995-6, 2005). Nous nous contenterons ici de retracer l'origine de l'institution rabbinique de la traduction biblique dans la liturgie synagogale, telle qu'elle est décrite et perçue dans la tradition juive. Nous nous appuierons sur des textes dont certains sont bien connus, mais qui ont été essentiellement étudiés par les théologiens ou les historiens. Nous souhaitons en proposer une synthèse et leur donner un éclairage spécifique, celui du traductologue.

\section{La Bible hébraïque ou Tanakh}

Le Tanakh (acronyme hébraïque formé des initiales des trois parties qui le constituent: Torah, Neviim, Ketouvim) est composé de 24 livres classés en trois groupes, (38 ou 39 unités textuelles). Son écriture s'est échelonnée sur près d'un millénaire. Sa division en trois parties est connue dès l'Antiquité. Le petit fils de Josué/Jésus Ben Sira les cite dans l'ordre dans le prologue de sa traduction du Siracide, l'œuvre de son grand-père, qu'il traduisit de l'hébreu

4. Directeur de l'académie talmudique de Soura, Sa'adia Gaon naquit en Haute Egypte, à Dilaz, en 882 ou 884 et mourut à Soura, en Irak, en 942. 
en grec à l'intention de ses coreligionnaires établis «à l'étranger»: «Torah, Prophètes et le reste des Écrits". Il prend garde de bien marquer la prééminence de l'original biblique sur la traduction:

Vous êtes donc invités à faire la lecture avec bienveillance et attention et à avoir de l'indulgence pour les passages où, semble-t-il, malgré le zèle de la traduction, nous n'avons pas été de force en certaines de nos tournures. Car les textes n'ont pas une force égale lorsqu'ils sont dits dans leur langue originale, en hébreu, et lorsqu'ils sont traduits dans une autre langue; et non seulement ce livre-ci, mais encore la Loi elle-même, les Prophéties et le reste des Livres offrent une supériorité qui n'est pas médiocre lorsqu'ils sont dits dans leur langue originale ${ }^{5}$.

À cette époque (II siècle av. J.-C.), La Sagesse de Ben Sira est encore considérée comme un livre sacré et l'entreprise de traduction écrite du Tanakh en grec a commencé un siècle plus tôt. Lorsque le canon de la Bible hébraïque sera fixé au Concile de Yavné (ou Jamnia, entre 90 et 110), certains des livres exclus du Tanakh (dont le Siracide) seront conservés dans le canon chrétien sous le nom de Pseudépigraphes ou Deutérocanoniques (en hébreu: Sefarim 'Hitsoniim, littéralement: Livres extérieurs).

\subsection{La Torah en soixante-dix langues}

Tous les textes du Tanakh n'ont pas le même statut au regard de la tradition juive. Certains sont considérés comme révélés, d'autres comme inspirés par l'esprit divin. Le corpus le plus sacré en est la Torah ${ }^{6}$ (littéralement: l'enseignement, l'instruction). Il est constitué de cinq parties, les "Cinq Livres de Moïse", 'hamicha 'houmechey Torah ou 'Houmach, le "Pentateuque». Mais le parchemin rituel calligraphié, lu à la synagogue, se présente comme un rouleau d'un seul tenant. La Torah est attribuée à Moïse, à qui Dieu parlait «face à face, comme un homme parle à son prochain" (Ex 33,11) et même "bouche à bouche» (Nb 12,8). La tradition affirme qu'en recevant la Torah, Moïse reçut en même temps son interprétation orale de la bouche même de Dieu au sommet du Mont Sinaï. Mais le peuple entier, massé au pied de la montagne, entendit la voix de

5. Le prologue du traducteur du Siracide est cité et analysé par Dorival (1988, 86-89).

6. Il arrive que l'on appelle Torah (au sens large) ce qui constitue le Tanakh tout entier, appelé aussi «Torah écrite» (Torah chébikhtav, littéralement: 'enseignement qui est dans l'écrit'), par opposition à la «Torah orale » Torah chébe'alpé: 'enseignement qui est sur la bouche'). 
Dieu énoncer le décalogue et plusieurs midrachim assurent que cette révélation était polysémique et polyglotte:

Il est écrit: «Le peuple entier voyait les voix » (Ex 20,15); le mot 'voix' est au pluriel. Rabbi Yo'hanan disait que toute voix sortait et se fractionnait (nè'hèlak) en sept voix et ces sept voix en soixante-dix langages (lachone) pour que toutes les nations entendent et que chaque nation entende Sa voix dans sa propre langue ${ }^{7}$.

Selon le Tanakh, Moïse lui-même mit par écrit le texte de la Torah (Dt 31,9), ordonnant à Josué de le lire tous les sept ans devant le peuple réuni (Dt 31,11-12). En passant le Jourdain, le peuple devait le recopier sur des pierres, en l'expliquant bien: baèr heytèv (Dt 27,3.8). Le livre de Josué affirme que ceci fut fait, que Josué recopia la Torah de Moïse (Jos 8,32) et qu'il en lut le texte en présence de tout Israël « et des étrangers qui vivaient parmi eux» (Jos 8,33.35).

Ce qui nous importe pour notre propos, c'est le commentaire que donnèrent les principaux exégètes juifs de ces textes. Pour eux, l'un des sens du baèr heytèv est que le texte doit être accompagné de son corollaire implicite, le bèour: une "clarification" pour ceux qui ne comprennent pas le sens du texte, une "traduction » pour les étrangers présents qui ne comprennent pas la langue du texte: "Vous écrirez sur les pierres toutes les paroles de cette Torah: écrit, gravé et bien expliqué, se lisant dans une langue et se traduisant dans 70 langues. » (Targoum Yonathan et TB Sota 36a. Nous traduisons). Le grand exégète champenois Rachi (1040-1105) choisit également cette interprétation du baèr heytèv de Dt 27,8: "bien clairement - en 70 langues ", ce qui correspond à l'interprétation qu'il avait donnée de la même expression figurant au premier chapitre du Deutéronome, lorsque Moïse avait commencé au pays de Moab d'expliquer la Torah devant tout Israël, baèr heytèv (Dt 1,5): "C'est en 70 langues qu'il la leur expliqua " (Rachi, nous traduisons).

\subsection{L'institution du targoum oral}

Selon la tradition juive, c'est Esdras le Scribe qui, sous le règne d'Artaxerxès, autour de 400-380 av. J.-C., aurait institué en Judée le rituel

7. Tan'houma sur Ex, simane 25, parag. 5 (nous traduisons). Voir aussi Sifré sur Dt Vezote Haberakha : «Lorsque Le Saint Béni-soit-Il se révéla pour donner la Torah à Israël, il ne se révéla pas dans une seule langue, mais dans quatre: l'hébreu (Sinaï), le latin (Séir), l'arabe (Paran) et l'araméen (rivevote kodèch)». 
de la lecture publique de la Torah, les lundis et jeudis — jours de marché - ainsi que le Chabbat (samedi) et les jours de fêtes. En effet, après le second retour en Judée des exilés de Babylone (grâce à l'Édit de Cyrus, 538 av. J.-C.), la plupart des rapatriés avaient oublié leur tradition et leur langue. Ils ne parlaient plus que l'araméen (la langue diplomatique et véhiculaire de l'Empire perse). Certains avaient épousé «des femmes d'Achdod, de Moab ou d'Amon. Leurs fils parlaient à moitié l'achdodi ou la langue de tel ou tel peuple, mais ne savaient pas parler le judéen" (Ne 13,23-24). Esdras fit donc accompagner la lecture publique du texte hébreu de sa traduction et de son interprétation orale en araméen. Il voulait que le peuple entier se réapproprie la Torah et comprenne le sens des versets lus, y compris les femmes, les enfants et les hommes ignorants. Près du lecteur se tenaient donc les lévites et les mévinim, ceux qui faisaient «comprendre» le texte: "Ils lisaient dans le livre, dans la Torah de l'Eternel, meforach, de telle sorte que l'on puisse comprendre la lecture. » (Ne 8,8). L'adjectif meforach signifie: expliqué, interprété de manière explicite. Selon Rab, un amora (maître) babylonien du III $^{\mathrm{e}}$ siècle, le verset suggère que le peuple comprenait la lecture en hébreu parce qu'elle était rendue explicite par sa traduction. Ainsi, peut-on lire dans le traité Nedarim:

Rab a dit: Que signifie: «Ils lisaient dans le livre de la loi de Dieu, d'une manière distincte, et ils en indiquaient le sens, pour faire comprendre ce qu'ils avaient lu » ? Le livre de la loi de Dieu, c'est le Texte lui-même; d'une manière distincte: c'est à dire en le traduisant; et ils en indiquaient le sens: allusion à la division en versets; pour faire comprendre ce qu'ils avaient lu: allusion à la ponctuation; certains disent qu'il s'agit du texte traditionnel (TB Nedarim 37b $)^{8}$.

Lorsqu'on sait que le texte de la Torah (et du Tanakh tout entier) est purement consonantique et linéaire, sans ponctuation ni vocalisation, sans chapitres ni titres, qu'il ne comporte que très peu d'espaces signifiants, on comprend que cette "traduction» était également une énonciation tenant compte d'une tradition de «lecture » dont le Talmud dit qu'elle a été reçue par Moïse en même temps que le texte consonantique: 'Hananya ben A'hi Rabbi Yehochoua disait: "Entre deux paroles [Dibbour de Dieu écrites

8. Trad. Elkaïm-Sartre, 1982, 680. La traductrice traduit le pluriel massorote/les traditions, par «texte traditionnel », induisant qu'il s'agissait de la version qu'on appellera plus tard "massorétique », texte découpé, ponctué et vocalisé conformément aux traditions orales de lecture par les massorètes, entre les vi ${ }^{\mathrm{e}}$ et vii ${ }^{\mathrm{e}}$ siècles. Voir aussi TB Meguilla 3b et TJ Meguilla 1, 2. 
sur les Tables d'Alliance, au Sinaii] il y a les nuances et les allusions [littéralement: la syntaxe et les lettres ${ }^{9}$ ] de la Torah, «fourrées» de Tarchich, [des interprétations de la Torah orale] comme l'océan ${ }^{10}{ }^{\text {» }}$.

Le Talmud fait en conséquence remonter à Esdras l'institution d'une traduction orale en langue vernaculaire, appelée plus tard targoum, qui accompagne obligatoirement, verset par verset, la lecture synagogale du texte de la Torah, le clarifie et le développe. Avec le temps, le targoum araméen fit partie intégrante de l'enseignement et de l'étude de la Torah dans les écoles et les académies talmudiques ${ }^{11}$. Le traité Berakhote 8 a attribue à deux amoraïm du III siècle, Rav Houna bar Yehouda (Babylonie) et Rabbi Ami (Palestine), l'habitude pour chaque juif d'étudier les passages bibliques lus en public durant la semaine «deux fois dans le texte et une fois dans le targoum $^{12}$ » (nous traduisons). Lorsque l'araméen cessa d'être compris, le targoum traditionnel fut accompagné ou remplacé par la version arabe de Sa'adia Gaon en Orient, par une traduction en langue vernaculaire puis par le commentaire de Rachi, verset par verset, dans les autres aires linguistiques. "Coprésents ${ }^{13}{ }^{\text {» }}$, original et traduction/interprétation se présentaient alors comme un couple obligé, l'un conservant la forme polysémique de l'original hébraïque, l'autre actualisant le sens en fonction du public-cible. En tout cas, la pratique qui consiste à accompagner d'une traduction orale la lecture synagogale de la section biblique du jour (pratique toujours en

9. Il y a 'jeu de mots' car dikdouk désigne à la fois la grammaire, les règles contraignantes de la langue, et les détails précis (ledakdek = entrer dans les détails, être précis), tandis que le pluriel otiyote désigne les lettres de l'alphabet. Ote au singulier signifie à la fois la lettre, le signe, le signal, l'annonce...

10. TJ Cheqalim 6,1 - chap. 6, page 49, col. 4, halacha 1 , nous traduisons. C'est ainsi que la tradition retrace l'origine de la "Torah orale ", plus tard consignée dans «l'océan du Talmud». (Celui de Jérusalem est arrêté au IV siècle, celui de Babylone vers l'an 500). La métaphore de "l'océan" indique l'immensité et la structure 'ouverte' du Talmud: entre chaque «vague» de parole, Dieu expliquait et précisait son propos à Moïse, demandant à ce dernier de transmettre (oralement aussi) cet enseignement au peuple d'Israël (Exode Rabba 47 et Tan'houma sur Ki Tissa).

11. Cf. Shinan 1993, 20. Il cite notamment Massekheth Soferim 16, 6 et Avoth de Rabbi Nathan recension B, chap. 12).

12. La formule hébraïque est : chnayim mikra veè'had targoum, deux fois dans l'original et une fois en traduction. Rachi explique qu'il s'agit du T. Onkelos (voir section 2.2).

13. Nous avons proposé le concept de "coprésence » dans notre article Kaufmann (2002). Nous appelions la traductologie à prendre en compte les cas où la traduction ne prétend pas être le texte lui-même, remplaçant un original absent, masqué, oblitéré. Figurant aux côtés de l'original, une traduction "coprésente » dialogue avec le textesource dans une altérité marquée et une complémentarité féconde et respectueuse. 
usage chez les Yéménites) est attestée dans les communautés de la diaspora de l'Antiquité et elle se maintiendra jusqu'au Moyen-Âge. Maïmonide (1138-1204) l'évoque ainsi: "La coutume qui s'est instaurée depuis l'époque d'Esdras veut qu'un interprète traduise pour le peuple ce que le lecteur lit dans la Torah, afin que le peuple comprenne le sens des choses (iniyane hadevarim)» (Michné Torah, Sefer Ahava, chap. 12, halakha 10; nous traduisons). Rachi disait que "le targoum s'adresse aux femmes et aux ignorants qui ne comprennent pas l'hébreu" (commentaire sur Meguilla 21b). Selon les circonstances, la traduction peut être littérale, paraphrastique, voire constituer une amplification ou plus rarement une omission délibérée. Le style en est généralement clair, le niveau de langue est celui de la langue courante, mais il arrive que la syntaxe soit irrégulière, la «coprésence » de l'original obligeant parfois l'interprète à suivre l'ordre hébraïque des mots. Les chercheurs ont montré que le targoum s'est développé parallèlement au Midrach aggada, l'exégèse homilétique, qu'il s'en nourrit quand il ne la précède pas (par ex. Shinan 1993).

\subsection{La signification du mot targoum}

Il faut éviter de se méprendre en pensant que targoum renvoie nécessairement à «traduction». En effet, les passages de la Bible dont l'original est araméen (dans Esdras ou Daniel) sont aussi appelés targoum, tout comme la traduction araméenne de la Bible. Dans le Talmud, targoum (sans autre précision) peut donc être un synonyme de «texte en araméen » (original ou traduction), alors que pour toute autre traduction, la langue est précisée ou bien l'on parle de traduction en n'importe quelle langue: «targoum bekhol lachone». Par ailleurs, targoum peut signifier «traduction intralinguistique », reformulation qui explique, précise, développe un texte peu accessible par d'autres mots de la même langue ${ }^{14}$. La racine du mot targoum a sans doute pénétré dans l'hébreu par l'araméen. Elle apparaît une seule fois dans la Bible, au livre d'Esdras 4,7: une missive est envoyée au roi de Perse : katouv aramite oumetourgam aramite, "écrite en écriture araméenne et traduite en araméen» (trad. Chouraqui ${ }^{15}$ ). Dans le Talmud, «traduire» mais aussi "interpréter" se dit: le-targuème et traducteur: "metourguemane» ou «tourguemane». On remarquera que dans les langues européennes (italien,

14. Dans le Talmud, «(Untel) tirguèm » signifie souvent : (untel) expliquait ainsi...

15. La King James traduit: "written in the Syrian tongue, and interpreted in the Syrian tongue». 
anglais, français, où le mot s'est introduit au Moyen-Âge par l'intermédiaire du grec de Byzance: dragoumanos et de l'arabe, d'où «drogman » puis «truchement »), le mot signifie essentiellement «interprète ", c'est-à-dire traducteur «oral », intermédiaire, ce qui correspond bien à la profession ou à la fonction du metourguemane (figure familière de la vie synagogale mais aussi des tribunaux et des académies de l'antiquité juive). Son targoum de la Bible est donc bien une interprétation orale, une "actualisation» du texte lu qui tient compte du public-cible, de l'époque et du lieu concernés ${ }^{16}$.

\subsection{Les Prophètes et la traduction de la Haftara}

Revenons au Tanakh. Si la «Torah de Moïse» est considérée comme d'origine divine, le second corpus de la Bible hébraïque est constitué d'écrits inspirés (Neviim: «Les Prophètes ») dont les recueils les plus tardifs sont attribués à Aggée, Zacharie et Malachie (prophètes contemporains du retour d'exil: $\mathrm{VI}^{\mathrm{e}}$ et $\mathrm{V}^{\mathrm{e}}$ siècles av. J.-C. ${ }^{17}$ ). Une partie de ces textes jouit néanmoins d'un statut rituel, puisqu'on les lit le Chabbat ou les jours de fête à la synagogue. La tradition juive la plus commune fait remonter cette pratique à l'époque des persécutions d'Antiochus IV Épiphane (175-164 av. J.-C.). Le roi séleucide aurait interdit la lecture publique de la Torah et les rabbins auraient substitué à la section sabbatique un passage parallèle des Prophètes appelé: Haftara (littéralement: supplément). Quelle que soit l'origine de cette coutume, elle s'est préservée au cours des siècles. La Haftara était également traduite oralement dans la lecture publique (en général par segments de trois versets : voir TB Meguilla 24a). Nous en avons un témoignage tardif dans un responsum émanant du Maharam de Padoue, Rabbi Méir ben Yits'hak Katzenellenbogen (1473 ? -1563 ? $)^{18}$, qui faisait office de président

16. Sur le metourguemane qui accompagne la lecture de la Torah dans le rite synagogal, nous ne nous étendrons pas ici mais renvoyons le lecteur à notre article: Kaufmann 2005, disponible en ligne à l'adresse : <http ://www.erudit.org/revue/meta/2005/v50/ n3/011608ar.html>

17. Bien que considéré comme "prophétique ", le livre de Daniel, de composition plus tardive, figure dans les Hagiographes.

18. Cf. Responsa du Maharam de Padoue, simane 78, dans le CD édité (en hébreu et araméen) par l'université Bar Ilan, Ramat Gan. Pour plus de détails sur cette base de données, on peut consulter le site du "Projet Responsa" à l'adresse: <www.responsa. biu.ac.il>. Ce texte du Maharam figure dans le mémoire de M.A. de Samy Grinberg, rédigé sous ma direction et soumis en 2007 à l'université Bar Ilan: The Translation in Jewish Responsa. The Attitude of Rabbis and Codifiers (Poskim) to the Translation of the Holy Scriptures (heb.). Sur le grec, voir aussi ci-dessous, note 25. 
du tribunal rabbinique de la République de Venise. Un juif italien lui demandait s'il fallait maintenir la coutume de lire à Kippour le livre de Jonas uniquement en traduction grecque (!), après avoir d'abord lu les trois premiers versets dans l'original hébraïque et avant de lire trois versets de Michée en hébreu puis de poursuivre la lecture du prophète en traduction grecque. Le Maharam répond qu'il ne faut pas annuler une coutume ancestrale. Il invoque notamment le statut particulier du grec dans la tradition de traduction orale synagogale et, pour justifier sa décision, il s'appuie sur le Talmud de Jérusalem qui prévoit qu'on peut se contenter d'une lecture minimale de trois versets de la Haftara en hébreu ${ }^{19}$ lorsqu'on fait appel à un metourguemane, un traducteur-interprète.

\subsection{Les Hagiographes}

Le troisième et dernier corpus, les Hagiographes (Ketouvim, littéralement: (les) Écrits), comprend notamment Les Psaumes et les Cinq rouleaux. Une partie de ces textes fait partie intégrante de la liturgie et déjà Soferim 14,1 mentionnait la bénédiction qu'il faut prononcer en lisant publiquement l'un ou l'autre des Cinq rouleaux «Béni sois-tu Eternel notre Dieu qui nous as sanctifiés par tes commandements et nous as prescrit de lire dans les Ecrits saints " (Kitevey Hakodèch; nous traduisons). C'est ainsi que vingtcinq Psaumes sont récités au cours de l'un ou l'autre des trois offices quotidiens. Le Cantique des Cantiques est lu le Chabbat et aux offices de Pessa'b (la Pâque), Ruth à Chavouoth (Pentecôte), l'Ecclésiaste à Souccoth (fête des Cabanes), Esther à Pourim, Les lamentations de Jérémie et Job lors du jeûne du $9 a v$, anniversaire de la destruction du Temple, sans compter des extraits de Daniel, Esdras, Nébémie et des Chroniques qui figurent dans l'office du matin (en plus de textes de la Torah). On connaît des targoumim araméens de la plupart de ces textes, destinés sans doute à l'origine à accompagner oralement la lecture synagogale des jours de fêtes en guise de haftara puis mis par écrit. Notons que pour le rouleau d'Esther, le Talmud de Babylone fait une exception. Quand l'assistance ne comprend pas l'hébreu, il autorise une lecture synagogale directement dans la langue «maternelle» du public: copte, élamite, mède, grec, etc. (TB Meguilla 18a).

19. «Rav énonçait : la Haftara se composera d'au moins vingt et un versets, lorsqu'il n’y a pas d'interprète; mais lorsqu'il y en a un, il peut suffire de lire trois versets. » (TJ Meguilla 4, nous traduisons). 
Dans ce cas uniquement, la lecture exclusive de la traduction a valeur rituelle (Michna Meguilla 2,1 $)^{20}$.

\section{Contrôle et accréditation des traductions}

La plupart des chercheurs admettent aujourd'hui l'exactitude de la tradition talmudique qui fait remonter à l'action d'Esdras l'institution d'une lecture publique de la Torah accompagnée de sa traduction en araméen et de son interprétation, autour de 400-380 av. J.-C. C'est dire que la plupart des targoumim écrits que nous connaissons s'appuient sur d'anciennes traditions interprétatives qui se transmettaient oralement de maître à disciple, donnant lieu parfois à des interprétations contradictoires. Il est probable qu'après une première période de relative liberté, le traducteur ait cessé d'improviser pour se conformer à une interprétation reçue, au point d'être repris, voire réprimandé si sa version ne correspondait pas à la tradition locale, comme l'attestent des anecdotes de l'époque talmudique. "Un tourguemane qui «officie» devant un Sage n'a pas le droit de retrancher, d'ajouter ou de changer, sauf si ce Sage est son père ou son Maître" (Tossefta Meguilla 3,41, nous traduisons). Signalons à ce propos que des ajouts, des omissions ou des «détournements» de sens n'étaient pas rares dans l'Antiquité, mais qu'à l'époque talmudique, ils furent condamnés, voire sanctionnés. La Michna Meguilla 4,9, rapporte ce qu'encourt celui qui traduit le verset (pourtant polysémique) concernant les sacrifices humains à Moloch (Lv 18,21), comme s'il concernait les unions interdites (la «fécondation " d'une femme araméenne ${ }^{21}$ ), "on le fait taire en lui infligeant un blâme (nezifa)» (sept jours d'éloignement). On faisait taire (sans blâme) celui qui utilisait des euphémismes intempestifs pour parler des unions illicites (supprimant ainsi la possibilité d'identifier l'acte interdit) ou celui qui donnait des interprétations hérétiques en ajoutant des mots ne se trouvant pas dans le texte.

Concernant les conflits d'interprétation, la Genèse qualifie les yeux de Léa, sœur aînée de Rachel, de «rakote» (doux, délicats, faibles, mouillés ?). Les uns voient dans cet adjectif un compliment, les autres une critique: «Le metourguemane de Rabbi Yossey traduisait ainsi :

20. Le cas particulier d'Esther s'explique par le fait que Mardochée avait envoyé des lettres aux Juifs des 127 provinces du royaume, rédigées dans la langue et dans l'alphabet de chaque destinataire (Est 8,10).

21. Voir Michna (1976), note 20, p. 21 sur Meguilla 9. Le verset permet un double jeu de mots sur zera' = semence ou descendance, et sur leha'avir le- $:$ ici, offrir ou engrosser. 
"Que signifie rakote? Ses yeux étaient humides». Rabbi Yo'hanan lui dit: «Tu n'as pas bien traduit. Que signifie rakote? Ses yeux étaient aroukote, allongés» " (Tan’houma, édition Buber, Vayétsé 20, nous traduisons). Il existe une variante de ce texte, beaucoup plus violente, qui témoigne non seulement du contrôle exercé sur l'établissement du «sens », du « vouloir dire » du texte, mais aussi des relations souvent conflictuelles entre les maitres (les Talmidey 'hakhamime, l'aristocratie intellectuelle de l'époque) et les professions auxiliaires représentées notamment par les lecteurs et les interprètes. Concernant ce même verset de la Genèse, Rabbi Yo'hanan reprit son propre interprète en l'insultant: "[Larmoyants? !]. Ce sont les yeux de ta mère qui sont larmoyants! Que signifie rakote? Ses yeux étaient mouillés de pleurs car elle pleurait en disant: que ce soit la volonté divine que je ne sois pas donnée à l'impie Esaü» (Genèse Rabba, 70,16, nous traduisons).

\subsection{Deux traductions écrites "autorisées"}

Devant ces conflits d'interprétation et certaines dérives, il importait d'uniformiser les diverses traditions de lecture. C'est à quoi s'employèrent, s'il faut en croire le témoignage du Talmud, deux maitres palestiniens du tout début du II siècle, Rabbi Eliézer [ben Hyrcan] et Rabbi Yehochoua [ben 'Hananya]. Elèves à Yavné de Yo'hanan ben Zakaï, connus pour leurs controverses fréquentes, ils sont pourtant associés pour revendiquer l'accréditation de deux traductions écrites «autorisées" du Pentateuque. Elles auraient été effectuées sous leur autorité par deux prosélytes, chacun traduisant vers sa langue maternelle: une traduction araméenne, recueillie et fixée par Onkelos (selon le Talmud de Babylone, Meguilla 3a) et une traduction grecque rédigée par Aquila (selon le Talmud de Jérusalem, Meguilla $1,71 \mathrm{c}$; cette dernière était destinée à remplacer la LXX dans l'usage juif).

Cette tradition rabbinique a été remise en cause par les chercheurs: on considère généralement qu'Onkelos et Aquila (dont les prénoms sont très proches) ne sont qu'une seule et même personne. Seul Aquila aurait existé, Onkelos étant le nom donné à un texte reçu. Chacun des deux Talmuds aurait voulu «canoniser» la traduction écrite qui avait le plus d'autorité et d'usage dans sa région: la traduction en araméen (dite derabanane, "de nos maîtres ", attribuée ultérieurement à Onkelos) s’imposant dans la zone située au nord de la Palestine et la traduction en grec d'Aquila prévalant au sud.

Ces traductions ont été abondamment étudiées par les chercheurs. Nous nous contenterons ici d'attirer l'attention sur la tradition rabbinique qui souligne que les deux traductions «autorisées » sont le fait de deux 
prosélytes. Les légendes les concernant abondent dans le Talmud. Attirés par le message de la Bible hébraïque, ils font profiter leurs nouveaux coreligionnaires de leur maîtrise de la langue et de la culture correspondant à la langue d'arrivée du texte diffusé. En revanche, leur compréhension de la langue de départ (l'hébreu), et leur interprétation du texte du Pentatenque sont garanties par l'autorité des deux maîtres de Palestine qui les légitiment. Onkelos aurait bénéficié d'une tradition de bouche à oreille: «Rabbi Yirmeya, selon d'autres Rabbi 'Hiya bar Abba, a dit: le Targoum sur la Torah, c'est Onkelos le prosélyte qui l'a «dit», tel qu'il l'avait reçu de (ses maîtres) Rabbi Eliezer et Rabbi Yehochoua » (TB Meguilla 3a, trad. Salzer 1978, $8^{22}$ ). Cette légende semble confirmée par la recherche qui veut que T.O. soit un targoum reflétant la tradition palestinienne. Il a été "importé » en Babylonie (en même temps que la Michna et la Tossefta), où il a été révisé et fixé au second siècle.

Pour Aquila, en qui la légende voit un proche parent de l'empereur Hadrien (qui régna de 117 à 138), le Talmud de Jérusalem emploie une autre formule qui suggère une transmission plus directe, de maître à élève $\mathrm{e}^{23}$ : «Rabbi Yirmeya, Rabbi 'Hiya bar Abba disaient: Aquila le prosélyte a traduit (tirguèm) la Torah, devant (lifeney) Rabbi Eliézer et Rabbi Yehochoua qui l'ont couvert de louanges. (TJ Meguilla 1,71c, nous traduisons). Le texte crée un jeu de mots qui met en relation le prénom d'Aquila et le verbe «couvrir de louanges» (le-kaless): «Rabbi Eliézer et Rabbi Yehochoua le glorifièrent (kilessou) en lui disant: "Tu l'as emporté en beauté (yafyafita) sur tous les hommes ${ }^{24}$ (TJ Meguilla 1,71, col. 3, hal. 9 ; nous traduisons).

À cette époque, le grec est encore considéré comme une langue prestigieuse et riche, convenant à la traduction de l'original hébraïque: la Guemara s'appuie sur la réussite d'Aquila pour argumenter que, contrairement à ce que dit la Michna, la Torah ne peut pas être traduite dans n'importe quelle

22. Comparer avec la traduction d'Elkaïm-Sartre $(1982,499)$ : Onkelos le prosélyte fit une traduction de la Torah sous la dictée de R. Éléazar et R. Josué ». Or le texte emploie le verbe amar [Onkelos a « dit» son targoum], ainsi que la formule mipi: littéralement "de la bouche" des deux rabbins (le sens est sans doute: en conformité avec leur enseignement; voir ci-dessous, section 2.3 : Jonathan ben Ouziel reçoit son targoum mipi, de la bouche de prophètes morts cinq siècles avant lui).

23. Rabbi Eliézer fut aussi le maître de Rabbi Akiva, dont la méthode exégétique aurait influencé Aquila.

24. Il y a ici un nouveau jeu de mots, cette fois sur la racine indiquant le beau (yafé), mise en relation avec le nom de l'ancêtre des Grecs: Yafet (Japhet). 
langue, mais seulement en grec: «Car après examen, on a constaté que la Torah ne peut être traduite de manière vraiment satisfaisante qu'en grec » (TJ Meguilla, ibid.; notre traduction).

Les rabbins appuient leur jugement sur une interprétation allégorique de Bar Kappara: lorsque Noé bénit son fils Japhet, il annonce que «Japhet résidera dans les tentes de Sem» $(\mathrm{Gn} 9,27)^{25}$. Il est permis sinon recommandé de traduire la Torah en grec, de faire résider la langue de Japhet dans l'enceinte du monde de Sem, de la Torah. C'est d'ailleurs à ce même enseignement que s'était référé Rabban Siméon ben Gamliel, (l'un des maîtres incontestés du judaïsme palestinien au milieu du premier siècle et grand admirateur de la langue grecque), pour proposer la règle halakhique qui veut que la seule langue étrangère autorisée pour la traduction de l'ensemble du Tanakh soit le grec (TB Meguilla 9b26).

Naomi Seidman $(2006,93)$ a raison de faire remarquer qu'en légitimant la résidence de Japhet chez Sem, les Rabbins mettent l'accent sur l'importation des beautés inhérentes à une culture étrangère pour enrichir la culture de l'original, contrairement à la direction habituelle des «transferts» linguistiques (transport, déplacement du texte source exporté vers l'étranger). La traduction de la Bible en grec est considérée comme un "gain" pour le texte source, écrit-elle, non comme une perte. Sem profite des beautés de Japhet (et la culture juive s'enrichit des qualités de la langue

25. Voir TB Meguilla 9b et Gn Rabba sur paracha 36 (trad. 1987, 376-77) et sur Deutéronome, paracha 1. Lire aussi le commentaire de Rabbi Obadia de Bertinoro (1440-1530), sur la Michna Meguilla, chap. 1, michna 8: "(Les Livres sacrés) s'écrivent en toute langue - dans l'alphabet (l'écriture) de chaque nation et dans l'idiome de chaque nation. Ils ne s'écrivent [c.-à-d. ne se traduisent] qu'en grec — la raison pour laquelle les Livres (du Tanakh) peuvent s'interpréter en grec plus qu'en toute autre langue se trouve dans le texte de la Genèse (chap. 9) «Dieu mettra Japhet au large [yaft, compris ici comme: 'embellira Japhet'] et il résidera dans les tentes de Sem», c'est yafyafouto (la beauté) de Japhet, c'est-à-dire le langage de toute beauté que parlent tous les fils de Japhet, qui résidera dans les tentes de Sem, or chez les descendants de Japhet l'on ne trouve aucune langue plus belle que le grec. La halakha suit l'avis de Rabban Siméon ben Gamliel. Néanmoins, à notre époque, cette langue grecque s'est corrompue, c'est pourquoi, l'on n'écrit les Livres (du Tanakh) à notre époque que dans l'écriture du sacré et dans la langue du sacré [uniquement en langue et en caractères hébraïques et non en grec] » (notre traduction).

26. Son avis l'avait emporté sur celui de Rabbi Juda bar Ilaï qui voulait aussi limiter la langue de traduction au seul grec, mais uniquement pour le Pentateuque, en se fondant sur le précédent de la Septante. Le Talmud raconte que le père de Siméon, Rabban Gamliel, avait mille élèves dont la moitié étudiaient la Torah et l'autre moitié la sagesse grecque (voir TB Baba Kama 83a et Sota 49b). 
et de la culture hellénistiques), mais en les "convertissant » à l'hébraïsme, de même qu'Aquila est devenu juif. Seidman va jusqu'à affirmer que ceci explique la méthode littérale adoptée par Aquila: «Le corps grec hébraïsé d'Aquila le prosélyte se reflète en miroir dans le texte grec hébraïsé qu'il produit. [...] Dans son adhésion aux principes herméneutiques d'Akiva et dans son incorporation de mots hébraïques à l'intérieur du texte grec, Aquila construit une Bible grecque dans laquelle l'hébreu n'est pas transcendé mais reproduit. » (ibid., nous traduisons).

De fait, conformément à l'approche de rabbi Akiva (qui enseignait qu'aucun élément formel ou sémantique du texte hébraïque n'était contingent et qu'il fallait interpréter chaque signe comme signifiant), Aquila retraduisit la Torah en fonction de l'état du texte hébraïque fixé à son époque, avec pour objectif manifeste la possibilité de proposer un texte grec susceptible de servir de support à l'étude et à l'exégèse juives. Son littéralisme délibéré permet de suivre l'original en le décryptant, segment par segment. Ce que d'aucuns appellent traduction «étymologique» (souci de faire toujours correspondre une même racine grecque à une même racine hébraïque) pourrait aussi n'être qu'un respect du principe tannaïtique de la guezèra chava, une sorte d'analyse intertextuelle avant la lettre, qui s'appuie sur la récurrence d'un même concept à travers le corpus biblique pour éclairer son sens et enrichir la signification de l'un de ses contextes d'occurrence par sa mise en parallèle avec un ou plusieurs autres contextes.

Par la suite, l'usage du grec s'étant perdu dans la plupart des communautés juives, le targoum d'Aquila a été oublié, tandis que l'araméen restait la langue de l'étude du Talmud, du Zohar et de certaines prières (dont le kaddich), conservant à la version dite d'Onkelos une place privilégiée dans l'étude de la Torah.

\subsection{L'autorité du Targoum Onkelos sur la Torah}

Onkelos se présente comme un double araméen qui accompagne l'original dont il offre une lecture conforme à la tradition rabbinique. Il lui arrive de proposer des paraphrases ou de tenter d'expliquer une règle, mais en général, il suit de très près la forme syntaxique et respecte le vocabulaire de l'original, énoncé ou lu en même temps que lui. Néanmoins, il supprime les anthropomorphismes, interprétés comme des métaphores, et adoucit les formulations trop crues.

Onkelos cessa très vite d'être utilisé dans le rituel de Palestine où l'on ne comprenait déjà plus son dialecte et où l'on préférait, semble-t-il, une 
interprétation plus libre. Mais il fit florès comme source d'exégèse «autorisée ». Le Talmud, Rachi, Maïmonide et l'ensemble de l'herméneutique juive recourent largement à Onkelos pour élucider des points controversés ou pour étayer une tradition de lecture. Parlant des conditions que prétend remplir un fiancé digne d'une femme de bon lignage, Juda bar Ilaï (rabbin né en Galilée, disciple de Rabbi Akiva, milieu du second siècle) expliquait dans une baraïta ${ }^{27}$ qu'il ne suffit pas que le prétendant sache lire au moins trois versets du texte de la Torah, il doit aussi être capable de les «interpréter». Ce tirgoum («traduction-explication») ne doit pas être «improvisé » (littéralement: de son propre cru, selon son opinion), mais il doit s'appuyer sur une solide connaissance de la tradition antérieure ou, comme le dit Rabbi Juda dans un aphorisme célèbre: "Celui qui traduit un verset littéralement (ketsourato: selon sa forme) est un affabulateur (badai); celui qui ajoute au texte est un impudent (me'harèf) et un moqueur (megadèf). Qu'est-ce que [savoir] traduire ? C'est «notre» traduction ${ }^{28} »$ (celle d'Onkelos selon Rachi et Maïmonide). Les disciples de Rachi, les Tossaphistes (XII ${ }^{\text {e }}$ siècle), s'appuyant sur un développement de TB Kiddouchine 49a expliquaient: "Celui qui traduit littéralement le verset: «Ils virent le Dieu d'Israël» (Ex 24,10) est un affabulateur, puisqu'on ne peut «voir» vraiment la Présence divine, comme il est écrit : "Car l'homme ne peut me voir et rester vivant» (Ex 33,0). Mais celui qui incorpore des ajouts en traduisant: "Ils virent l'ange de Dieu», c'est un moqueur puisqu'il attribue à un ange la gloire de Dieu» (nous traduisons). Les Tossaphistes comprennent donc le verset selon l'exégèse d'Onkelos : voir Dieu, c'est ici voir «la gloire du Dieu d'Israël».

Rachi considère que les interprétations d'Onkelos ne sont ni arbitraires ni fantaisistes parce qu'elles s'enracinent dans la tradition orale, reçue au Mont Sinaï, et qu'il a su s'écarter du mot à mot afin de clarifier les difficultés du texte.

27. Une baraïta est un texte hébraïque de Terre sainte datant au plus tard du $\mathrm{II}^{\mathrm{e}}$ siècle mais non retenu par le compilateur de la Michna, Rabbi Juda Hanassi, parce qu'exprimant une opinion minoritaire. Intégrant les baraïtote dans la Guemara (close vers l'an 500), les Maîtres de Babylonie s'y réfèrent dans leurs discussions.

28. TB Kiddouchine 49a, nous traduisons. Le même texte se trouve dans Tossefta Meguilla 3 (4), 41, sans le mot megadef. De fait les substantifs me'haref et megadef sont souvent énoncés ensemble et se renforcent. On peut aussi les comprendre comme: injurieux et insultant. Ou encore : querelleur et railleur; ou même: sacrilège et blasphémateur. Notons que badai, que nous traduisons par "affabulateur» (bedaya = fantaisie, imagination) est traduit par certains comme: "menteur" (en hébreu: chakrane). Sur cet aphorisme, voir Shinan 1993, 25. 
Depuis l'invention de l'imprimerie, le Targoum Onkelos a été composé en regard et sur la même page que l'original hébraïque de la Torah, qu'il accompagne (jusqu'à ce jour). Nous avons vu que c'est Onkelos que lisent aujourd'hui encore les Yéménites, (avec la traduction arabe de Sa'adia Gaon), pour étudier la section sabbatique de la semaine, tout comme l'ont fait jusqu'au Moyen-Âge l'ensemble des communautés juives.

Notons que la version «autorisée » d'Onkelos n'est pas le seul targoum araméen sur le Pentateuque. On connaissait déjà bien le Targoum Yiroushalmi, ou Targoum de Jérusalem, longtemps attribué par erreur à Jonathan ben Ouziel (et appelé pour cela Pseudo-Jonathan). Rédigé dans un araméen caractéristique du parler des Juifs de Galilée, il a trouvé une forme définitive autour du VII ${ }^{\mathrm{e}}$ ou du VIII ${ }^{\mathrm{e}}$ siècle. La découverte en 1930 d'un manuscrit connu sous le nom de «Codex Neofiti I a fait progresser la connaissance des targoumim en livrant un troisième texte, probablement mis par écrit en Galilée entre le VII et le IX ${ }^{\mathrm{e}}$ siècle. Sans compter le Targoum Yiroushalmi II (ou "Targoum fragmentaire ») et d'autres segments retrouvés dans la Gueniza du Caire et ailleurs. Pourtant, seul Onkelos continue d'être étudié et cité jusqu'à ce jour comme texte vivant.

\subsection{L'accréditation du Targoum Yonathan sur les Prophètes et l'interdiction des targoumim sur les Hagiographes}

Nous avons vu que des passages des Prophètes étaient lus et traduits dans le rituel synagogal depuis l'époque hasmonéenne. Rédigés dans un style poétique ou métaphorique, les textes prophétiques exigeaient d'être interprétés pour révéler leur sens véritable. Là encore, une version écrite " autorisée » s'est imposée: le Targoum Yonathan, rattachée non plus à une autorité rabbinique, mais attribuée à l'inspiration directe des derniers prophètes et donc considérée comme une traduction "révélée ". Issu de Palestine, ce targoum a lui aussi été "importé » par les académies de Babylone et a subi de nombreuses révisions avant d'adopter une forme définitive au VII siècle. Comme celui d'Onkelos, il est fréquemment appelé à la rescousse dans la littérature rabbinique pour élucider des passages prophétiques qui, sans lui, seraient devenus incompréhensibles (ex.: Moed Katane 28b, Sanhedrin 94b, Meguilla 3a). Comme Onkelos, il s'écarte des anthropomorphismes, mais il est moins littéral, plus paraphrastique, surtout dans les segments poétiques ou dans les Derniers Prophètes (notamment Isaïe, Jérémie, Michée) où il lui arrive d'amplifier le texte en recourant à des traditions «aggadiques» (légendaires). 
Le même passage du traité Meguilla qui parle de l'accréditation rabbinique du Targoum d'Onkelos sur la Torah évoque les circonstances miraculeuses de la traduction araméenne des Prophètes:

La traduction araméenne (le targoum) des Prophètes, c'est Jonathan ben Ouziel qui l'a énoncée de la bouche de Aggée, Zacharie et Malachie; la Terre d'Israël trembla alors sur quatre cents pars $a^{29}$ de large et quatre cents parsa de long. Une voix céleste se fit entendre: "Qui a révélé Mes secrets aux fils de l'homme? " Jonathan ben Ouziel se dressa et dit: «C’est moi qui ai révélé Tes secrets aux humains; mais Toi tu sais bien que je ne l'ai pas fait pour ma gloire, ni pour la gloire de la maison de mon père, mais c'est pour Ta propre gloire que je l'ai fait, afin que les polémiques ne se multiplient pas en Israël ». Il [Jonathan ben Ouziel] voulut encore révéler la traduction araméenne (Targoum) des Hagiographes mais une voix céleste lui dit: "C'en est assez! ». Pour quelle raison? Parce qu'on y trouve [les secrets de] la venue du Messie (TB Meguilla, 3a, nous traduisons).

Ce texte ne prétend pas exprimer une vérité historique, puisque Jonathan ben Ouziel a vécu environ cinq siècles après les prophètes Aggée, Zacharie et Malachie. Cet anachronisme se résout si l'on comprend qu'il a recueilli la tradition orale reçue et transmise de maître à élève depuis l'époque des prophètes, et qu'il l'a harmonisée et fixée. Mais l'essentiel est ailleurs.

La raison invoquée ici pour justifier l'établissement d'une version " autorisée », conforme à l'enseignement des auteurs inspirés, est la crainte de scissions idéologiques internes au peuple juif. Et l'on voit apparaître une réticence à partager avec tout un chacun les «secrets ", la tradition ésotérique (contenue notamment dans les visions apocalyptiques de Daniel). Ce thème d'une transmission réservée aux initiés apparaît également au niveau de l'étude de l'original (ex.: TB 'Haguiga 13a; Gn Rabba 8,2), mais elle est plus rigoureuse encore pour l'usage des traductions qui, par définition, s'adressent à des étrangers ou à des juifs qu'on qualifierait aujourd'hui d' "assimilés ", qui ne sont plus capables d'accéder au texte original parce qu'ils ont perdu le contact, non seulement avec leur langue, mais aussi avec leur culture et avec les traditions qui ont porté le texte révélé. (C'est sans doute la raison pour laquelle certains textes peuvent être lus mais non traduits à la synagogue, voir TB Megilla 25a-b). En conséquence,

29. Une parsa $=$ un parasange $=$ entre 4 et 4,6 km. Voir Steinsaltz 1994, 254-255: «Poids et mesures ». 
les mêmes rabbins qui louent la traduction de la Torah énoncée par le prosélyte Onkelos et tolèrent une traduction orale des Prophètes, reçue « de la bouche » même des derniers prophètes par un disciple juif du grand Hillel, s'opposent (dans le texte cité) à une traduction des Hagiographes (incluant Daniel).

Pourtant, si les targoumim d'Onkelos et de Jonathan ont fini par être légitimement mis par écrit et uniformisés, nous savons que des targoumim écrits des Hagiographes ont tout de même existé dès l'Antiquité, notamment sur Job et les Psaumes. Un targoum de Job a été retrouvé parmi les manuscrits de la mer Morte. Or le Talmud parle d'un targoum de Job retiré de la circulation par Gamliel Ier, contemporain de Hillel - début du $\mathrm{I}^{\text {er }}$ siècle (voir TB Chabbat 115a). Serait-ce l'œuvre de Jonathan ben Ouziel qui, dans notre texte de Meguilla, évoque son désir d'entamer un targoum des Hagiographes et se fait rabrouer? De fait, chacun des Hagiographes a donné lieu à une ou plusieurs versions araméennes. Certaines sont citées dans le Talmud et les sources rabbiniques. Parfois littérales, souvent paraphrastiques (Esther, Ecclésiaste), voire allégoriques (Cantique des Cantiques), elles se caractérisent par des approches et des styles différents qui exigeraient une étude minutieuse au cas par cas.

\section{La Septante au regard de la tradition juive}

\subsection{La Septante dans la littérature non rabbinique}

Jusqu'à présent, nous avons essentiellement évoqué les traductions orales qui se sont peu à peu figées dans l'écrit. L'histoire de la Septante est différente. Le grec était parlé en Judée depuis sa conquête par Alexandre le Grand (323 av. J.-C.), ainsi que par les juifs exilés en Egypte ${ }^{30}$ et par les nombreuses communautés hellénistiques établies sur le pourtour méditerranéen. La plupart avaient oublié l'usage de l'hébreu sans pour autant parler la langue locale. C'est ainsi que le grec finit par se substituer insensiblement à l'hébreu dans leur pratique rituelle (lecture synagogale, prière, inscriptions funéraires).

C'est dans ce contexte que naquit la décision de produire une traduction écrite de la Torah en grec. Deux traditions s'affrontent à cet égard:

30. Si l'on en croit les historiens, aux alentours de l'ère chrétienne, la communauté juive d'Egypte était forte de deux cents mille âmes (dont la moitié à Alexandrie). On trouve de nombreuses anecdotes dans le Talmud qui attestent de la magnificence de la synagogue d'Alexandrie (ex. Soucca 51b) et de la richesse de la communauté. 
l'une affirme que cette traduction a été entreprise à l'initiative de la communauté juive pour soutenir ses efforts de diffusion des valeurs juives dans une société où la polémique philosophique était âpre, et où les sympathisants et les prosélytes venus au judaïsme étaient de plus en plus nombreux. La LXX devait également constituer un texte de référence pour remplacer à la synagogue les traductions orales en grec effectuées par les metourguemanim et jugées imparfaites.

Une seconde tradition attribue à l'initiative d'un souverain la décision de faire traduire les livres de Moïse en grec. La légende (partiellement acceptée par les historiens ${ }^{31}$ ) veut que ce soit le roi Ptolémée II Philadelphe (285246 av. J.-C.) qui, à l'initiative de son bibliothécaire, Démétrios de Phalère (qui était pourtant tombé en disgrâce sous Philadelphe), passe commande d'une traduction grecque de la Torah pour enrichir la Bibliothèque d'Alexandrie. Quoi qu'il en soit, la LXX n'obtint jamais le statut de traduction autorisée qui sera accordé cinq siècles plus tard au Pentatenque d'Aquila. Mais des sources juives non rabbiniques font état de l'enthousiasme de la communauté d'Alexandrie à son égard, notamment la Lettre d'Aristée (deuxième siècle av. J.-C., voir Lettre d'Aristée 1962, 58; Dorival 1988, 40), largement reprise par deux auteurs juifs du Ir siècle, Philon d'Alexandrie et Flavius Josèphe. Ces auteurs nous donnent un aperçu précieux sur le statut du traducteur et sur leur conception de la traduction des textes sacrés. Leurs versions ne concordent pas toujours. Le pseudo-Aristée insiste sur la considération dont jouissent les traducteurs, choisis par le grand prêtre de Jérusalem: "Eléazar choisit les hommes du plus grand mérite, d'excellente éducation, vu la distinction même de leurs parents, et qui étaient passés maîtres dans les lettres judaïques, mais s'étaient de plus, adonnés sérieusement à la culture hellénique » (Lettre d'Aristée 1962, 121122). En traduisant, ils se mettent d'accord entre eux sur chaque point de confrontation (ibid., 301-302, et 231). La communauté juive reçoit cette œuvre commune avec une allégresse non dépourvue de crainte révérencielle. En effet, à l'issue d'une lecture publique de la LXX, Aristée rapporte cette mise en garde des traducteurs:

31. Sur la discussion de la légende et les hypothèses faisant de la Septante une traduction de substitution à l'initiative des juifs assimilés ou un targoum à usage rituel, ou encore une «commande» royale pour compléter la collection d'ouvrages célèbres traduits pour la Bibliothèque d'Alexandrie, lire notamment Le Déaut, Perrot et de Ward 1984, ainsi que Dorival 1988. 
Maintenant que la traduction a été faite correctement, avec piété et avec une exactitude rigoureuse, il est bon que cette œuvre reste comme elle est, sans retouche $[. .$.$] À ce mot, ce fut une acclamation générale { }^{32}$ : alors ils les invitèrent à prononcer une malédiction, selon leur usage, contre quiconque retoucherait la lettre du texte soit en l'allongeant, soit en l'altérant si peu que ce fût, soit en y retranchant; excellente mesure pour le garder à jamais immuable ${ }^{33}$.

Nous retrouvons ici plusieurs principes entrevus dans l'aire araméophone, appliqués cette fois à la traduction: nul ne doit ajouter, retrancher, ni rien changer au texte "sacré ». La personnalité du traducteur est également essentielle: les Septante sont des juifs pieux et scrupuleux. La traduction se fait sous une autorité reconnue: ici la caution du Grand prêtre qui les a choisis est gage de leur intégrité. On insiste également sur leur double culture et sur leur connaissance des deux langues concernées (leur langue maternelle serait celle de l'original). Remarquons que cette première traduction écrite - près de cinq siècles avant la version écrite d'Onkelos est transcrite dans un alphabet non sémitique, ce qui permet d'emblée une lecture aussi bien par des non-juifs que par des juifs. L'auteur de la Lettre d'Aristée met d'ailleurs en garde contre une utilisation «littéraire» de la parole divine. Selon lui, les historiens et les poètes païens qui, avant les LXX, avaient tenté d'insérer dans leurs œuvres des citations traduites de la Bible hébraïque, avaient été "frappés d'un châtiment ", " dû à l'indiscrétion d'avoir voulu livrer les choses divines à des profanes » (235-237). Le thème des secrets divins, qu'il ne faut pas révéler aux non-initiés et aux païens apparaît donc très tôt, avant même le récit talmudique sur le targoum de Jonathan ben Ouziel.

Philon d'Alexandrie, dit aussi Philon le Juif (20 av. J.-C.- 47 ?), établit lui aussi, dans La Vie de Moïse (2,25-44), une équivalence entre le Tanakh et sa traduction grecque, la LXX, qu'il présente comme une œuvre «inspirée » par l'esprit divin. Il considère les traducteurs comme " hiérophantes ${ }^{34}$ et prophètes ", employant tous, spontanément, les mêmes mots et les mêmes tournures, "comme sous la dictée d'un invisible souffleur ». Philon décrit même la fête (à laquelle il dit avoir assisté) au cours de laquelle juifs et

32. Ibid., 233, note 2. Cette «acclamation» explique l'instauration de la fête de Pharos.

33. Ibid., 235 et 234-235, note 1, sur les imprécations contre les tentatives de correction du targoum grec.

34. Littéralement: qui révèlent (les mystères) sacrés. De hieros «sacré » et phainein « révéler ». 
non-juifs célèbrent chaque année l'anniversaire de la traduction miraculeuse et rendent grâces à Dieu de son bienfait dans l'île de Pharos ${ }^{35}$. À son époque - tout comme dans le récit de son contemporain Flavius Josèphe - la LXX est considérée par la communauté des Juifs d'Egypte comme une traduction «inspirée».

\subsection{La Septante dans la littérature rabbinique}

La littérature rabbinique se fait l'écho de ce statut privilégié. On en trouve un exemple dans une double baraïta du Talmud de Babylone. Lorsque l'ensemble des rabbins de la Michna sont d'avis qu'il est permis de traduire la Bible dans n'importe quelle langue, que Rabban Siméon ben Gamliel pense que seul le grec est autorisé à cause de la prophétie de Noé, Rabbi Juda bar Ilaï (milieu du II siècle, auteur de l'aphorisme cité ci-dessus, section 2.2) voudrait quant à lui limiter cette autorisation à la traduction, en grec, des seuls « Cinq Livres de Moïse », sur la foi du précédent de la Septante. Mais son opinion restrictive n'est pas retenue:

Une baraïta enseigne: Rabbi Juda dit: même quand nos maîtres ont autorisé l'écriture des Livres en grec, ils ne l'ont autorisé que pour le Pentatenque. Et cela à cause de l'épisode du roi Ptolémée. Une baraïta rapporte en effet que le roi Ptolémée réunit 72 Anciens, les installa dans 72 maisonnettes sans leur révéler la raison pour laquelle il les avait réunis. Puis il se rendit chez chacun d'eux et il leur dit : écrivez-moi [en grec] la Torah de votre maître Moïse. Le Saint-béni-soit-Il inspira chacun de telle sorte qu'ils aboutirent tous à la même pensée. (TB Meguilla 9a)

Selon cette tradition palestinienne, l'inspiration des 72 traducteurs est d'autant plus miraculeuse qu'isolés et au secret, ils aboutissent sans se concerter aux mêmes réflexions qui les poussent à reformuler certains versets susceptibles de prêter à confusion ou d'induire une lecture fautive ou malintentionnée, préjudiciable au bon renom du peuple d'Israël et de ses grands ancêtres $^{36}$. Ce que suggèrent le Talmud et le Midrach (car l'on retrouve des

35. L'île est célèbre aussi parce qu'elle abrite le Phare d'Alexandrie.

36. Là encore, nous ne nous étendrons pas et renvoyons le lecteur à notre article: Kaufmann 1990, ainsi qu'à la somme réunie par Harl et al. 1988. Lire tout ce passage de Meguilla en traduction française dans Aggadoth du Talmud de Babylone 1982, 502. 
variantes de cette légende dans de nombreux corpus rabbiniques ${ }^{37}$ ), ce n'est pas la similitude parfaite de l'ensemble du texte composé par chacun des traducteurs, mais c'est que chacun d'eux a introduit «sous l'inspiration divine» les mêmes modifications. Les études sur la LXX ont longtemps qualifié ces changements d'altérations délibérées, suggérant une manipulation théologique. Outre les problèmes d'évolution parallèle des deux corpus (le texte hébraïque qui aboutit au TM, texte massorétique, et les divers témoins de la LXX), on tend aujourd'hui à parler de différents procédés traductionnels, de techniques de transfert linguistique et culturel appliquées pour traduire le vouloir-dire du texte de la Torah ${ }^{38}$. Tenant compte des réflexes de lecture du public cible (Ptolémée et son peuple), les traducteurs auraient cependant fait usage d'un principe de précaution, évitant de reproduire certaines images ou formes syntaxiques que le lecteur de l'original comprend sans peine mais qui, traduites littéralement, risquent de suggérer une lecture polythéiste ou de laisser apparaître des incohérences.

Les chercheurs ont montré qu'il ne faut pas prendre les textes rabbiniques au pied de la lettre. Ce ne sont pas treize, ni sept, ni cinq mais des centaines de modifications traductologiques que les spécialistes ont relevées, tandis que des révisions tardives de la LXX ont supprimé quelquesunes des modifications citées dans Meguilla 9a et b.

Relisant le texte talmudique, nous apprenons surtout que, conscients des risques encourus en livrant à un regard étranger un texte destiné à la lecture interne, les Juifs de l'Antiquité reconnaissaient la nécessité d'adapter leur traduction en fonction du récepteur du texte et qu'ils considéraient ces modifications comme légitimes et «inspirées » par l'esprit divin.

Toutefois, cette approche contraire à certains principes fondamentaux (puisque les traducteurs ajoutent, retranchent et modifient des éléments de l'original, fût-ce sous l'inspiration du ciel $^{39}$ ), n'était pas recommandée par

37. Par exemple TJ Meguilla 1, p. 71, col. 4, hal. 9, qui parle de treize modifications, tout comme ce texte plus tardif: "Concernant le roi Ptolémée, on raconte qu'il réunit 72 sages, les installa dans 72 maisonnettes sans leur révéler pourquoi il les avait réunis. Il entra chez chacun d'eux et leur dit: écrivez-moi la Torah de votre maître Moïse. Dieu inspira chacun d'eux; ils aboutirent tous à une seule et même opinion et lui écrivirent la Torah elle-même. Mais ils modifièrent treize formulations que voici...» (Massekhet Soferim 1,7-8, nous traduisons). Voir aussi Gn Rabba, paracha 8 , sur deux ou trois des changements introduits.

38. Voir Soferim 1,8 (note précédente), qui affirme que le texte traduit était « la Torah ellemême».

39. C'est également la stratégie des targoumim, dont les ajouts ou les modifications sont interprétés a postériori comme «inspirés " par l'esprit divin. 
tous. Le Midrach Rabba, texte de la même époque (V siècle) reprend une grande partie des passages incriminés dans Meguilla. Évoquant lui aussi le risque de malentendu causé par le pluriel divin et par la formulation anthropomorphique dans le verset: "Faisons un homme à notre image, selon notre ressemblance» (Gn 1,26), le Midrach Rabba affirme qu'il ne faut rien modifier et que celui (des minim, des hérétiques) «qui veut se tromper qu'il se trompe $^{40} !$ ». Ce même passage avance que la lecture du contexte permet d'ailleurs de dissiper tout malentendu ${ }^{41}$, alors qu'une clarification excessive supprime une intention exprimée dans l'original. L'introduction du thème de la polémique religieuse entre Israël et les minim ${ }^{42}$, liée à l'interprétation et à la traduction du Tanakh, permet de comprendre l'évolution d'abord lente puis radicale qui fera de la LXX, d'abord considérée comme inspirée, voire « révélée ", une traduction prohibée.

\subsection{Le rejet de la Septante}

À ce stade de l'histoire de la traduction juive de la Bible, on ne discerne encore que peu de réticences devant l'acte de traduire la parole de Dieu. La méfiance s'installe et l'attitude s'inverse lorsqu'une âpre polémique s'engage entre juifs et chrétiens dans le monde hellénistique. L'Église adopte la Septante (plusieurs fois révisée) comme "Ancien Testament » et la considère comme seul texte authentique et inspiré (l'original hébraïque ayant été, à ses dires, altéré, censuré, et privé de toutes ses allusions à la venue du Christ). La controverse porte sur l'identité du véritable Israël. On ressent la profondeur de la blessure, côté juif, à la lecture de cet apologue midrachique, reproduit dans plusieurs recueils au VIII e et au IX siècle. Il y est dit que Moïse aurait demandé à Dieu l'autorisation de mettre par écrit non seulement la Torah mais aussi la Michna (la Torah orale). Mais Dieu aurait réservé l'interprétation orale à Israël:

Le Saint-béni-soit-Il avait prévu que les nations du monde traduiraient la Torah et la liraient en grec, disant : »Nous sommes Israël. Et jusqu'à présent

40. Gn Rabba, paracha 8. Nous traduisons. Voir traduction de tout le passage dans Midrach (1987), 110 (8); ve-harotsé lite'ote, yite'è est traduit par: "qui voudra se tromper se trompera ".

41. Ibid., 110-111 (9).

42. À l'origine, minim désigne toujours des juifs qui professent des opinions hérétiques. Peu à peu, le terme désigne aussi des non-juifs. Dans la Galilée du deuxième siècle, il désigne en priorité un non-juif. 
la balance est égale (entre les Juifs et nous) ». Le Saint-béni-soit-Il répliqua aux Nations du monde: "Vous vous dites mes fils, je n'en sais rien; seuls sont mes fils ceux qui possèdent mes secrets ». De quoi s'agit-il ? De la Michna qui fut donnée oralement (Midrach Tan’houma sur Ki Tissa, simane 34, Chemote Rabba 47 et Psikta Rabbati, 14 b, nous traduisons).

Notons le désaveu de la LXX, traduction grecque attribuée ici aux «Nations du monde» et non pas aux Juifs de Judée délégués à Alexandrie par le Grand Prêtre. Dépossédés de leur héritage de « fils de l'Alliance », les Juifs se referment et affirment que sans une tradition "authentique " de lecture, le texte sacré reste une lettre scellée pour ceux qui prétendent se l'approprier ${ }^{43}$. L'attitude vis-à-vis de la LXX et du grec s'inversent. La fête annuelle qui célébrait l'anniversaire de la traduction de la Septante sur l'île de Pharos devient un jeûne annuel ${ }^{44}$. Quant à l'affirmation du Talmud de Jérusalem selon laquelle "la Torah ne peut être traduite de manière vraiment satisfaisante qu'en grec », elle est renversée et contredite dans deux traités mineurs du Talmud (au contenu presque similaire), compilés tous deux au milieu du VIII siècle. On y affirme que, lorsque les Anciens "écrivirent la Torah en grec pour le roi Ptolémée, ce jour fut aussi dur pour Israël que le jour où le veau d'or fut fabriqué, puisque la Torah ne pouvait être traduite de manière vraiment satisfaisante " (Sefer Torah 1,8 et Massékhet Soferim 1,7, nous traduisons ${ }^{45}$ ).

Par ailleurs Soferim 1,7 juxtapose curieusement, sans les départager, deux traditions opposées: celle qui condamne la Septante (traduction attribuée

43. Lire à ce sujet Mena'hem Hirshman 1992, 16-17, chap. 1 .

44. Les commentaires rabbiniques contemporains affirment que la traduction aurait été imposée aux Juifs par un décret royal et non entreprise de leur plein gré. Le talmudiste Jacob Gordin (1896-1947) expliquait le jour de jeûne et de deuil lié à la LXX en disant que la traduction de la Bible est une entrée en galout, une entrée dans l'exil (Gordin 1995, p. 177-178). Mais Alpert (1998, 269, 2 $2^{\text {ème }}$ col.) considère que cette condamnation viendrait du fait que les juifs de la diaspora grecque et romaine utilisaient la LXX à la place de l'original et que la traduction aurait été réalisée à partir de manuscrits hébraïques non «standards".

N.B.: il ne faut pas confondre Meguillath Taanith (Le Rouleau des Jeûnes) et Massékhet Taanith (un traité du Talmud).

45. Les chercheurs pensent que ces traités mineurs ont reçu leur forme définitive à l'époque gaonique, même si leur origine est plus ancienne et remonterait à des traditions orales de l'époque tannaïtique (sans doute ii $^{\mathrm{e}}$ ou iii $^{\mathrm{e}}$ siècle). Concernant la Septante, nous pensons que la «censure » (ou la correction de bonne foi) opérée sur le texte favorable du Talmud de Jérusalem conduit à affirmer que la condamnation de la traduction grecque dans la version écrite définitive des traités concernés représente une évolution tardive. 
à cinq Sages juifs) et celle qui voit en elle un texte inspiré (Ptolémée disant à 72 Sages : "écrivez-moi la Torah de votre maître Moïse. Dieu inspira chacun d'eux; ils aboutirent tous à une seule et même opinion et lui écrivirent la Torah elle-même ", voir ci-dessus notes 36 et 37). Par ailleurs, tout en reflétant le rejet de la LXX pour des raisons théologiques évidentes, Soferim affirme l'importance, voire la nécessité de la traduction biblique à usage interne, pour le bon déroulement de la liturgie, puisque la compréhension du texte lu est primordiale: "Si (le lecteur) s'entend à traduire, c'est bien; sinon il le donne à traduire à quelqu'un qui s'y entend, afin que le reste du peuple, les femmes et les enfants comprennent; car il faut que les femmes comprennent ce qui est lu comme tout un chacun, à plus forte raison les hommes » (Soferim 18,4-5 $\left.{ }^{46}\right)$. Ce n'est donc pas l'entreprise de traduction biblique qui est remise en cause à partir du vIII ${ }^{\mathrm{e}}$ siècle, mais bien l'usage fait a posteriori de la seule Septante.

\section{Postface}

Il faut arrêter là ce tour d'horizon des textes concernant la traduction biblique dans l'antiquité juive. Nous espérons avoir montré qu'il n'existe pas de tabou juif contre la traduction ni d'obligation d'adopter la technique du mot à mot. Pourtant, l'usage fait par le monde non juif des traductions de la Bible hébraïque, notamment de la LXX, a induit une crainte et une méfiance qui ont fini par s'installer dans la littérature rabbinique. On constate par la suite une double tendance. La première s'inquiète du risque de «réduction » du message polysémique à un message unique et figé, et de la possibilité de déviances théologiques. Elle a suscité des réactions de fermeture et de dénigrement de la traduction, exigeant que l'étude de la parole divine soit réservée au peuple juif et ne passe que par un contact direct avec l'original hébraïque et avec l'exégèse rabbinique. On constate cependant une volonté parallèle d'offrir un large accès à la parole divine à tout être humain ${ }^{47}$. Sans se vouloir prosélyte, le courant juif « universaliste » affirme que la Torah a été donnée à l'ensemble de l'humanité et que sa traduction était prévue dès l'origine. Nous avons vu que, dans le sillage du Talmud, de nombreux midrachim affirment notamment que Dieu se

46. Ce passage concerne plus spécifiquement la lecture du Livre des Lamentations de Jérémie, durant le jeûne du 9 av.

47. Là encore, faute de pouvoir développer, nous renvoyons le lecteur à l'un de nos articles: Kaufmann (1995-6). 
révéla au mont Sinaï dans toutes les langues du monde pour que toutes les nations l'entendent (TB Chabbat 88b, midrach Tan'houma sur Ex, simane 25, parag. 5, Yalkoute Chimoni sur Jr, Remez 306, Sifré sur Dt Vezote Haberakha, etc.).

Dans l'usage interne, à la synagogue et dans les maisons d'étude, l'exigence de faire comprendre le texte hébraïque à tout un chacun (hommes, femmes, enfants, «étrangers » nouvellement convertis), et les affinités entre traduction et interprétation, poussèrent les rabbins à recommander que la traduction accompagne l'original comme un double "coprésent ", envisagé parfois comme un impératif imposé par la règle talmudique, parfois comme un mal nécessaire. Même aujourd'hui, où la traduction n'est plus admise dans le rituel synagogal (sauf dans les courants réformés ou dans le rite yéménite), elle est tolérée par certains, encouragée par d'autres, pour l'étude et l'enseignement juif, afin de perpétuer la transmission du message divin en respectant les traditions de lecture et d'exégèse. Nous en voulons pour preuve les nombreuses maisons d'édition juives d'obédience ultraorthodoxe, qui proposent aujourd'hui des traductions imprimées de la Bible (mais aussi des prières et des principales sources juives) où la traduction «en n'importe quelle langue » et les notes exégétiques encadrent l'original hébraïque.

On peut dire, en conclusion que la traduction du texte biblique, envisagée comme une facette légitime et utile à la compréhension du texte, est valorisée dans la tradition juive, tant qu'elle ne prétend pas se substituer à l'original.

\section{Références}

\section{Textes}

Aggadoth du Talmud de Babylone (la Source de Jacob - 'Ein Yaakov) (1982) / trad. et annoté par A. Elkaïm-Sartre, Paris, Verdier (Les Dix Paroles).

Lettre d'Aristée à Philocrate, (1962) / éd. et trad. par A. Pelletier, Paris, Cerf.

Midrach Rabba, Tome I, Genèse Rabba, (1987) / trad. par B. Maruani et A. Cohen-Arazi, Paris, Verdier (Les Dix Paroles).

(La) Michna, (1976): Meguila / trad. Par D. Gottlieb, Paris, Keren Hasefer ve-Halimoud.

(Le) Talmud de Babylone, la Guemara: Traité Meguilla (1978) / trad. par I. Salzer, Paris, Keren Hasefer ve-Halimoud et Colbo. 


\section{2. Études}

AlPERT, M. (1998), "Torah translation ", dans Routledge Encyclopedia of Translation Studies, M. BAKer, M. editor, London and New York, Routledge, p. 269-273.

Ballard, M. (1992), De Cicéron à Benjamin, Presses universitaires de Lille.

Berman, A. (1984), L'épreuve de l'étranger. Culture et traduction dans l'Allemagne romantique, Gallimard (Les Essais).

(1985), «La traduction et la lettre ou l'auberge du lointain » dans Les Tours de Babel, Gérard Granet éd., Mauvezin, Trans-Europ-Repress, p. 31-150.

Chouraqu, A. (1972), La Saga des Juifs d'Afrique du Nord, Paris, Hachette.

Gordin, J. (1995), Ecrits, Le renouveau de la pensée juive en France, Paris, Albin Michel (Présences du Judaïsme).

Harl, M., G. Dorival et O. Munnich (1988), La Bible grecque des Septante. Du judaïsme hellénistique au christianisme ancien, Paris, Cerf (Initiations au christianisme ancien).

Hirshman, M. (1992), Hamikra oumidrasho (Mikra and Midrash, A comparison of Rabbinics and Patristics), (hébreu) Tel-Aviv, Hakibbouts Hameou'had (Hillel ben-'Hayyim).

Kaufmann, F. (1990), «Un exemple d'approche théologique de la traduction: les jugements sur la Septante", dans La traduction des textes sacrés: le domaine biblique, TTR (Traduction, Terminologie, Rédaction), 3/2, p. 33-51.

(1995-6), «Le discours rabbinique sur la traduction juive de la Bible ", dans La traduction des textes sacrés, Koine, Anno V-VI, p. 283296.

(2002) «La coprésence de l'original et de sa traduction », dans F. IsRAËL, dir., Identité, altérité, équivalence? La traduction comme relation, Paris, Minard, p. 323-338.

(2005), «Contribution à l'histoire de l'interprétation consécutive: le Metourguemane dans les synagogues de l'Antiquité », dans G. BASTIN, dir., Le prisme de l'histoire, META, 50/3, p. 972-986.

Le Déaut, R. (1984), «La Septante, un Targum? », dans R. Kuntzmann et J. SCHLOSSER, dir., Études sur le judaïsme hellénistique (Congrès ACFEB 1983), Paris, Cerf (Lectio Divina 119), p. 147-195. 
Perrot, C. (1984), «La lecture de la Bible dans la Diaspora hellénistique », dans R. KUnTZMANN et J. SCHLOSSER, dir., Études sur le judaïsme hellénistique (Congrès ACFEB 1983), Paris, Cerf (Lectio Divina 119), p. 109132.

RobInson, D. (1996), Translation and Taboo, Dekalb Illinois.

SeIDman, N. (2006), Faithful Renderings: Jewish-Christian Differences and the Politics of Translation, Chicago, University of Chicago Press.

Shinan, A. (1983), "On the Nature of the Aramaic Targums to the Pentateuch ", Prooftexts, 3/1, p. 41-50.

(1993), Mikra E'had vetargoumim harbé (The Biblical Story as reflected in its Aramaic translations), (hébreu) Tel-Aviv, Hakibbouts Hameouéhad (Hillel ben-'Hayyim).

Steiner, G. (1975), After Babel. Aspects of Language and Translation. Oxford, Oxford University Press.

Steinsaltz, A. (1994), (Le) Talmud. Guide et lexiques / trad. française par les rabbins J.-J. Gugenheim et J. Grunewald, Paris, FSJU et JC Lattès.

WAARD, J. de (1984), «La Septante: une traduction », dans R. KunTZMANN et J. SChlosser, dir., Études sur le judaïsme hellénistique (Congrès ACFEB 1983), Paris, Cerf (Lectio Divina 119), p. 133-146.

\section{Résumé}

Un préjugé répandu voudrait qu'un tabou juif interdise de traduire la Bible ou n'autorise qu'une traduction servile, littérale. Cette étude s'appuie sur la littérature rabbinique (Talmud, Midrach, exégèse) pour montrer qu'une traduction orale à usage interne a été prescrite dès l'Antiquité, quand la majorité des Juifs ont perdu l'usage de l'hébreu. Sa fonction était d'accompagner — non de remplacer - l'original hébraïque, dans la lecture synagogale et dans l'étude. Elle a été mise plus tard par écrit puis imprimée en regard de l'original. La traduction qui s'enracine dans la tradition exégétique juive est légitime puisqu'elle contribue à clarifier et expliquer et le sens de la Bible hébraïque. 


\section{Abstract}

According to a widespread bias, there would be a Jewish taboo against the translation of the Bible, or at least a Jewish translation should be as close as possible to the letter of the original. This paper, based on the study of rabbinic literature (Talmud, Midrach, exegesis), intends to show that an oral translation, for internal purposes, was already needed in Antiquity, when most of the Jews could not understanding Hebrew anymore. Its function was not to replace but to be read along the Hebrew original in the synagogue and in the study. Later on, it has been written down and printed with the original text. Translation which is imbedded in the exegetic Jewish tradition is legitimate and contributes to understanding the meaning of the Hebrew Bible. 